\title{
The TIR Domain of TIR-NB-LRR Resistance Proteins Is a Signaling Domain Involved in Cell Death Induction
}

\author{
Michal R. Swiderski, Doris Birker, and Jonathan D. G. Jones \\ Sainsbury Laboratory, John Innes Centre, Norwich Research Park Colney Lane, Norwich, NR4 7UH, U.K.
}

Submitted 20 January 2008. Accepted 2 October 2008.

\begin{abstract}
In plants, the TIR (toll interleukin 1 receptor) domain is found almost exclusively in nucleotide-binding (NB) leucinerich repeat resistance proteins and their truncated homologs, and has been proposed to play a signaling role during resistance responses mediated by TIR containing $R$ proteins. Transient expression in Nicotiana benthamiana leaves of "TIR + 80", the RPS4 truncation without the NB-ARC domain, leads to EDS1-, SGT1-, and HSP90-dependent cell death. Transgenic Arabidopsis plants expressing the RPS4 $\mathbf{T I R}+\mathbf{8 0}$ from either dexamethasone or estradiol-inducible promoters display inducer-dependent cell death. Cell death is also elicited by transient expression of similarly truncated constructs from two other $R$ proteins, RPP1A and At4g19530, but is not elicited by similar constructs representing RPP2A and RPP2B proteins. Site-directed mutagenesis of the RPS4 TIR domain identified many lossof-function mutations but also revealed several gain-of function substitutions. Lack of cell death induction by the E160A substitution suggests that amino acids outside of the TIR domain contribute to cell death signaling in addition to the TIR domain itself. This is consistent with previous observations that the TIR domain itself is insufficient to induce cell death upon transient expression.
\end{abstract}

Additional keywords: apoptosis.

In animal and plant kingdoms, the TIR (Toll interleukin 1 receptor) domain is present in proteins involved in immune responses. In mammals, the TIR domain is an effector module in TOLL-like receptors (TLR), the interleukin-1 (IL-1) receptor family, and small adaptor proteins that link active TLR and IL1 receptors to downstream signaling components (Takeda and Akira 2005). In plants, the TIR domain has been recognized at the $\mathrm{N}$-terminus of resistance proteins carrying the nucleotide binding (NB)-ARC domain and leucine-rich repeats (LRR). Huge sequence diversity exists among NB-LRR proteins, and they confer resistance to various microbial pathogens, viruses, and invertebrates. The TIR-NB-LRR class of resistance $(R)$ genes in the Arabidopsis genome is represented by nearly 100 genes. In addition, there are more than 50 genes encoding proteins without either an LRR or NB-LRR portion. Intriguingly, the TIR domain is essentially absent from gramineae genomes

Current address for D. Birker: Max Planck Institute for Plant Breeding Research Carl-von-Linné-Weg 10, 50829 Köln, Germany.

Corresponding author: J. D. G. Jones; Fax: (0044) (0) 1603 450011; Email: jonathan.jones@sainsbury-laboratory.ac.uk

* The $\boldsymbol{e}$-Xtra logo stands for "electronic extra" and indicates that three supplemental figures are published online.
(Meyers et al. 2002, 2003). The other subclass of NB-LRR proteins either lacks a recognizable $\mathrm{N}$-terminal motif or carries a coiled-coil (CC) domain at the $\mathrm{N}$-terminus.

The NB-ARC domain comprises a region of extended homology between Apaf1, R proteins, and Ced4 (van der Biezen and Jones 1998a). It has been demonstrated that the NB-ARC domain from both the $\mathrm{I} 2$ resistance protein and Apaf1 exhibits ATPase activity in vitro (Riedl et al. 2005; Tameling et al. 2002, 2006). This implies that an energy-dependent conformational change in $\mathrm{R}$ proteins is crucial for their activity.

The LRR domain mediates protein-protein and proteinligand interactions and is involved in the specific recognition of pathogen-derived elicitors (Dangl and Jones 2001; Dodds et al. 2006; Hammond-Kosack and Jones 1997). The recognition of avirulence proteins is, at least in some instances, indirect and consistent with the "guard" hypothesis (van der Biezen and Jones 1998b; Dangl and Jones 2001). Proteolytic removal of RIN4 by the Pseudomonas syringae effector, AvrRpt2, activates RPS2, inducing defense and cell death (Axtell and Staskawicz 2003; Mackey et al. 2003). Similarly, the cleavage of PBS1 kinase by AvrPphB protease is essential for activation of the RPS5 resistance protein (Shao et al. 2003). Conceivably, many CONSTITUTIVE pathogenesis-related $(P R)$ gene expressor ( $c p r)$ mutations might be located in genes encoding putative "guardees." For example, a mutation in the BON1 gene which encodes a copine synthase induces defense responses by activating the SNC1 TIR-NB-LRR R protein (Yang and Hua 2004).

Activation of $\mathrm{R}$ proteins is likely to lead to a conformational change, as has been documented for the CC-type $\mathrm{R}$ proteins Rx and Bs2 (Leister et al. 2005; Moffett et al. 2002). In the presence of coat protein from Potato virus $X$, interactions between CC, NB, and LRR domains of Rx are disrupted (Moffett et. al. 2002; Rairdan and Moffet 2006). Similar intramolecular interactions have not been observed for TIR containing $\mathrm{R}$ proteins, although it has been reported that the $\mathrm{N}$ protein oligomerizes in the presence of its cognate elicitor (Mestre and Baulcombe 2006). Genetic requirements for the signal transduction pathways initiated by $\mathrm{CC}$ and TIR R proteins only partially overlap. Although the CC class seems to be dependent on NDR1, the TIR class is dependent on EDS1 (Aarts et al. 1998; Falk et al. 1999). Two factors known to be involved in protein folding, SGT1 and HSP90, are shared, at least partially, by both pathways (Azevedo et al. 2002; Bieri et al. 2004, Hubert et al. 2003; Takahashi et al. 2003; Tornero et al. 2002b; van Bentem et al. 2005).

It has been reported that elevated expression of $R$ genes in plants induces constitutive immunity and that the transient expression of $R$ genes may induce spontaneous cell death (Leister and Katagiri 2000; Oldroyd and Staskawicz 1998; Zhang et al. 2004). It seems that the TIR domain plays a crucial role in a 
cell death signaling pathway (Weaver et al. 2006; Zhang et al. 2004). Indeed, transient expression in tobacco leaves of the flax L10 TIR domain with extra C-terminal amino acids induces cell death (Frost et al. 2004). However, the role of the TIR domain is still poorly understood.

Here, we show that the NB-ARC domain is not necessary for induction of cell death caused by an expression of truncated derivatives of TIR resistance proteins. Expression of truncations containing the TIR domain with a short C-terminal fragment directly following it is sufficient to cause cell death. We analyze the TIR domain of the RPS4 protein by sitedirected mutagenesis and characterize several gain-of-function substitutions.
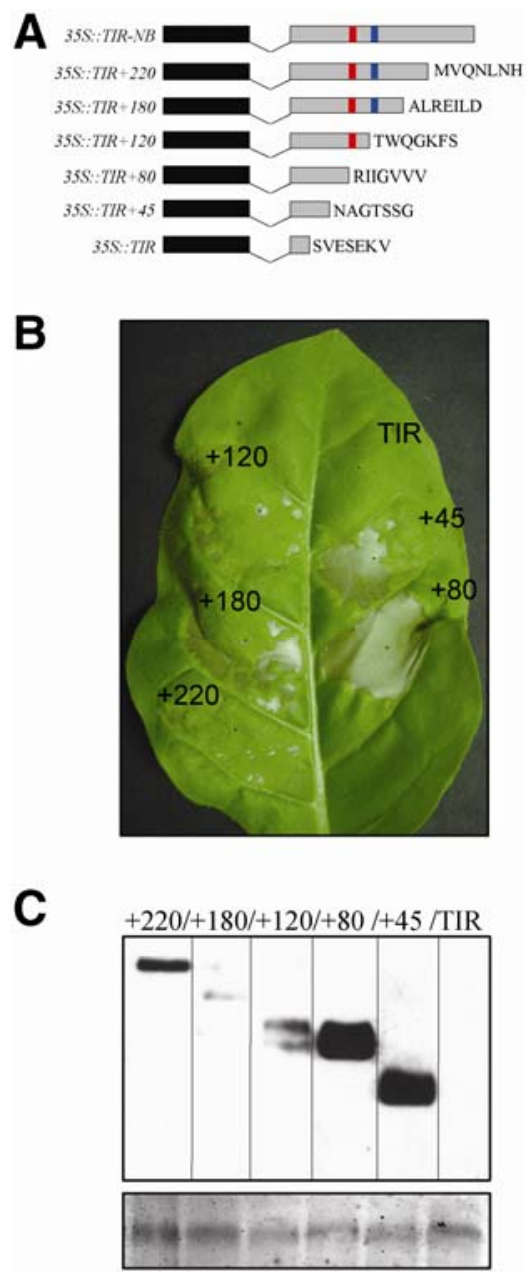

Fig. 1. Truncated derivatives of RPS4 without nucleotide-binding (NB)ARC domain induce cell death upon transient expression in Nicotiana tabacum leaves. A, Schematic representation of the deletion alleles of RPS4 used in B. TIR (toll interleukin 1 receptor)-NB-ARC (35S::TIR-NB) provides a comparison with other constructs. The TIR domain is shown as a black box. Expression of all derivatives is driven by the $35 \mathrm{~S}$ promoter of Cauliflower mosaic virus. Red and blue boxes mark Walker A and Walker B motifs present in the NB-ARC domain, respectively. Letters at the end of boxes represent amino acids at which particular construct ends. B, Derivatives of RPS4 as in A were delivered into different segments of a tobacco leaf via Agrobacterium. Cell death was observed 36 to $48 \mathrm{~h}$ later. Picture was taken 4 days postinfiltration. Construct expressing the TIR domain did not induce cell death. C, Expression of constructs delivered into tobacco leaf. Samples were taken 40 to $48 \mathrm{~h}$ after infiltration. The membrane was probed with an anti-hemagglutinin-HRP conjugated antibody. The TIR construct could not be detected. Equal protein loading was checked by staining membrane with Ponceau S (bottom panel). Visualized bands represent the large subunit of rubisco. All experiments were repeated a minimum of four times and produced a similar pattern of responses.

\section{RESULTS}

The NB-ARC domain of RPS4 is not necessary for induction of cell death.

Previous data suggest that the TIR domain of RPS4 is important for induction of cell death when RPS4 is transiently expressed in tobacco leaves (Zhang et al. 2004). However, this inference was based on the absence of cell death induction by RPS4 derivatives lacking their TIR domain. To investigate more directly a role for the TIR domain of RPS4, a series of constructs were made, deleted through the NB-ARC domain (Fig. 1A). The TIR+80 and TIR+45 constructs terminated before the NB motif. A single hemagglutinin (HA) tag was incorporated at the $3^{\prime}$ end of each truncated construct. Transient Agrobacterium spp.-mediated expression of these truncations in tobacco leaves allowed us to evaluate cell death induction. Surprisingly, all constructs except the one which encoded only

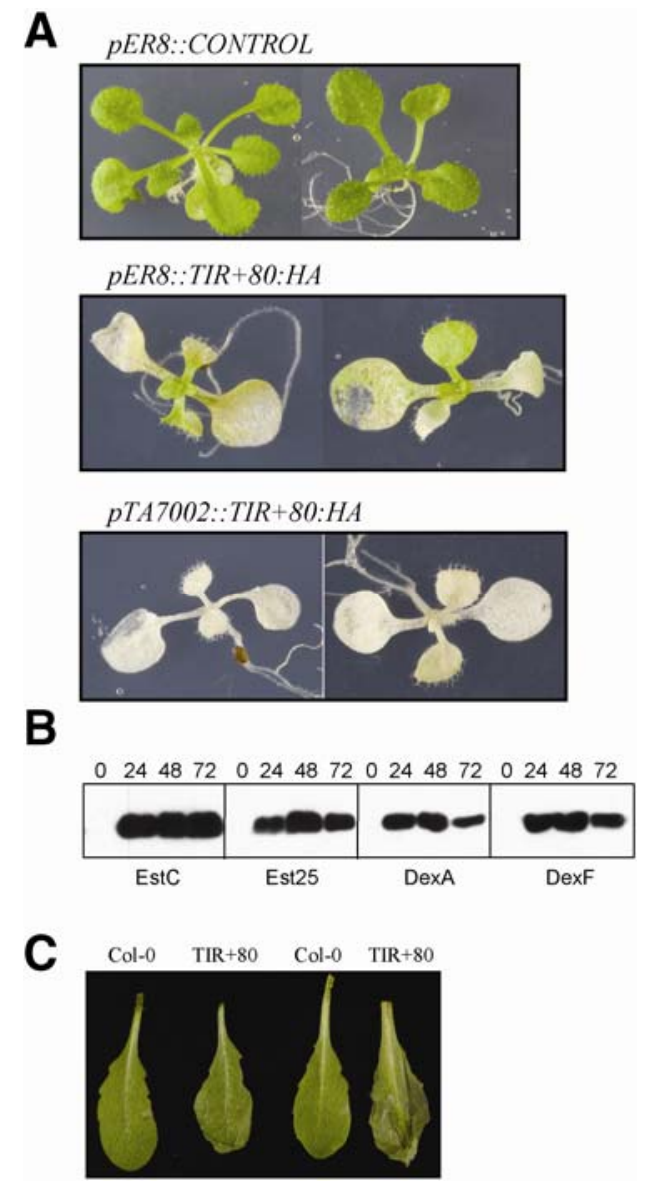

Fig. 2. TIR +80 induces cell death in Arabidopsis seedlings. A, pER8:: $T I R+80$ or pTA7002:TIR +80 transgenic seedlings of Arabidopsis Col-0 were transferred after 10 to 14 days to fresh medium supplemented with either $40 \mu \mathrm{M}$ estradiol or dexamethasone. The top pictures represent the control with unrelated fragment cloned into pER8 vector. Pictures in the middle represent seedlings after 5 days on estradiol-supplemented medium. Bottom pictures present seedlings expressing $T I R+80$ from pTA7002 vector. Pictures were taken 5 days after transplantation to induction medium. Control transgenic lines expressing FLAG22 peptide did not show any symptoms. B, Expression of TIR+80 after treatment with estradiol (Est) or dexamethasone (Dex). Proteins were extracted from seedlings at $0,24,48$, and $72 \mathrm{~h}$ after induction. Est and Dex names below represent independent transgenic lines transformed with estradiol- or dexamethasoneinducible construct, respectively. C, pTA7002::TIR+80 transgenic Arabidopsis exhibit cell death after infiltration of the dexamethasone (right side of a leaf). Cell death is visualized as collapsed leaf tissue. Picture taken 24 $\mathrm{h}$ after the dexamethasone infiltration. Similar results were obtained in three independent repeats. 
the TIR domain of RPS4 and a very short C-terminal extension (TIR) were able to cause cell death (Fig. 1B). This validated our previous conclusion that the TIR domain is indeed an effector domain for cell death signaling. Nearly all respective proteins were detected, though steady state accumulation of the individual proteins differed substantially (Fig. 1C). The inactive TIR construct produced an undetectable level of protein while the TIR +80 construct produced a high amount of protein.

To investigate whether Arabidopsis is also prone to cell death mediated by the expression of TIR +80 , the corresponding fragment was cloned into pTA7002 and pER8 vectors in which gene expression is controlled by the dexamethasone- or estradiol-inducible promoters, respectively (Aoyama and Chua 1997; Zuo et al. 2000). Both pTA7002::TIR+80:HA and pER8::TIR+80:HA were stably transformed into Arabidopsis and transgenic plants were tested for cell death upon induction. Young seedlings from several independent transgenic lines, grown on solid Murashige-Skoog (MS) medium in petri dishes, were transferred to fresh medium supplemented with either $40 \mu \mathrm{M}$ dexamethasone or estradiol. Three days later, most seedlings started to bleach and, after 5 days, most of them were dead (Fig. 2A). Similar data were obtained with both sets of transgenic lines, although the dexamethasoneinducible lines ( $p T A 7002:: T I R+80: H A)$ developed cell death faster than $p E R 8:: T I R+80: H A$ lines. Western blot analysis of protein extracts form both estradiol- and dexamethasone-challenged seedlings revealed that TIR+80:HA indeed accumulated in induced plants (Fig. 2B).

In addition, as an independent test to show that $\mathrm{TIR}+80$ induces cell death in Arabidopsis, the leaves of pTA7002:: TIR+ 80:HA transgenic lines were infiltrated with dexamethasone solution. Cell death was apparent $9 \mathrm{~h}$ later and, $17 \mathrm{~h}$ after infiltration, the whole leaf was usually dead (Fig. 2C).

\section{Cell death induced}

by $\mathrm{TIR}+80$ is $E D S 1, S G T 1$, and $H S P 90$ dependent.

We then tested whether the TIR+80-mediated cell death was dependent on EDS1, SGT1, or HSP90. All three genes have been shown to be important in pathogen-induced cell death and plant disease resistance (Jones and Takemoto 2004). $S G T 1, E D S 1$, and $H S P 90$ were silenced individually in Nicotiana benthamiana using virus-induced gene silencing (VIGS) (Zhang et al. 2004) and RPS4 TIR domain-dependent cell death was transiently induced in these plants. To assay cell death, the dexamethasone-inducible construct was used because it gave more reproducible results. In wild-type plants and TRV-00-silenced plants, cell death usually occurred $24 \mathrm{~h}$ after inducer application but, in EDS1-, SGT1-, and HSP90silenced plants, cell death either did not develop or was notably diminished compared with control plants (Fig. 3A). Similar results were obtained with the estradiol-inducible TIR+80 construct (not shown). Sampling protein extracts with an antiHA antibody showed that the TIR +80 protein accumulated equally well in SGT1-, EDS1-, HSP90-, and TRV-00-silenced plants (Fig. 3B).

\section{Truncated derivatives}

of two other TIR-NB-LRR proteins induce cell death.

We were interested to establish whether the cell-death-inducing property of TIR+80/TIR+45 was unique to the RPS4 protein or if homologous fragments from other TIR-NB-LRR genes could also cause cell death upon transient expression. TIR+80 fragments from $R P P I A, N$, and TIR+45 fragments from four genes from the RPP2 locus (At4g19500, At4g19510, $A t 4 g 19520$, and $A t 4 g 19530$ ) were constructed and tested via an Agrobacterium sp. expression system for their cell-deathinducing activity. At4g19500 and At4g19510 represent RPP2A and $2 B$, respectively (Sinapidou et al. 2004). Two constructs of the RPP1A TIR+80 fragment were prepared, with and without the N-terminal hydrophobic transmembrane peptide (Botella et al. 1998; Weaver et al. 2006). All fragments were fused to the single HA epitope. Both RPP1A constructs $(-\mathrm{H}$ and $+\mathrm{H})$ and the At4g19530 construct induced confluent cell death in the area of Agrobacterium sp. infiltration. The fragment from the $N$ gene induced only a weak, spotty cell death, whereas others were not active at all (Fig. 4A). Immunoprobing of protein
A

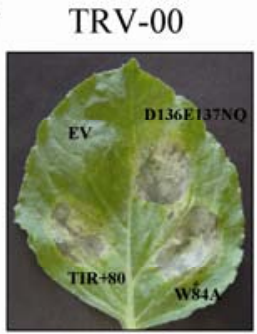

$5 / 6$

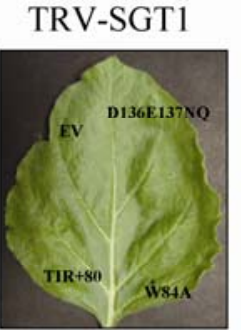

$2 / 15$

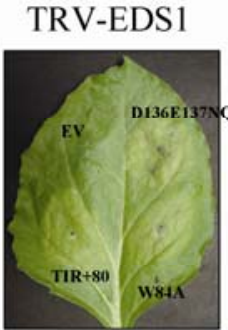

$2 / 10$

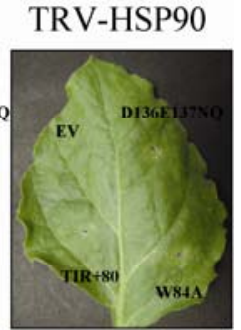

$3 / 8$

B

00 HSP90 EDS1 SGT1

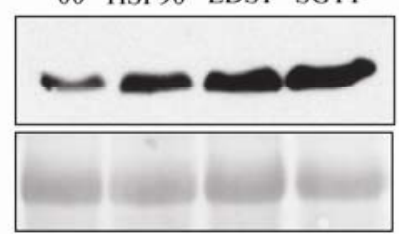

Fig. 3. Expression of the RPS4 TIR+80 derivative leads to EDS1-, SGT1-, and HSP90-dependent cell death. A, Nicotiana benthamiana plants were silenced using virus-induced gene silencing with indicated viral constructs. The dexamethasone-inducible TIR+80 derivative ( $p$ TA7002::TIR+80:HA), its two gain-offunction forms, or empty vector (EV) were delivered into plant cells via Agrobacterium spp. Two days later, dexamethasone was gently infiltrated into same areas on leaves. Cell death was scored $48 \mathrm{~h}$ later. Photographs recorded $96 \mathrm{~h}$ after dexamethasone infiltration. Numbers indicate the proportion of tested leaves that developed cell death in three independent experiments. A maximum of two leaves per silenced plant were tested. B, Expression of TIR+ 80 after induction with dexamethasone. Samples were taken $24 \mathrm{~h}$ after dexamethasone infiltration and protein blots probed with an anti-hemagglutinin-HRP conjugated antibody. Bottom panel shows Ponceau S staining of the membrane as a check of equal loading. Visible bands represent the large subunit of Rubisco. 
samples taken from these plants indicated that the lack of cell death induced by the TIR+45 derivative of At $4 \mathrm{~g} 19520$ and greatly diminished cell death caused by expression of $\mathrm{N}+80$ might be due to a much lower amount of protein accumulation compared with fragments from either RPS4 or TIR+80 fragments from other $\mathrm{R}$ proteins (Fig. 4C through E). TIR+45 fragments from At4g19500 and At4g19510 accumulate well; thus, lack of cell death induced by these proteins is most likely due to intrinsic properties of these proteins rather than due to insufficient expression level.

\section{Mutagenesis of the RPS4 TIR domain.}

To further characterize the RPS4 TIR domain, we introduced 32 mutations into the TIR+80 fragment. Amino acids chosen for site-directed mutagenesis were based on their conservation in an amino acid sequence alignment of plant TIR domains and, in most instances, were hydrophilic (Fig. 5; Supplementary Fig. 3; Table 1). Secondary structure prediction suggested a similar arrangement of $\beta$-sheets and $\alpha$-helices in plant TIR domains as in TIR domains from IL-1R and TLR1, although the $\beta$-strand $E$ is uncertain (Fig. 5). For some positions, multiple substitutions were constructed. These mutated TIR+80 fragments were tested for their ability to induce cell death. Cell death was visually evaluated based on the size of the infiltrated area that collapsed and the timing of cell death. Routinely, leaves were checked 24, 36, and $48 \mathrm{~h}$ after Agrobacterium $\mathrm{sp}$. infiltration. Both Table 1 and Supplementary Figure 1 summarize our observations of the cell death elicited by the mutated derivatives of TIR +80 .

All mutated proteins were detected, except mutations E111K and $\mathrm{E} 134 \mathrm{~K}$, and most of the substitutions accumulated to a level comparable with wild-type TIR +80 (Fig. 6). We noticed that several mutations, notably substitutions E111K and E134K, induced a faster and much stronger cell death response than the control (Table 1). The gain-of-function character of these mutations was additionally revealed by an Agrobacterium dilution and ion leakage assays. In the dilution assay, the Agrobacte- rium sp. harboring a construct was diluted to different concentrations and infiltrated into tobacco leaves. Agrobacterium strains carrying gain-of-function mutations in TIR +80 always induced cell death at the concentration 5 to 25 times lower than wild-type TIR+80. Comparisons of conductivity measurements taken from leaf samples expressing the gain-of-function mutant versions of TIR +80 , an inactive control (TIR), a wildtype TIR +80 , and one mutant version of TIR +80 which does not change cell death activity are shown in Figure 7. All gainof-function mutations produced 1.3 to 2 times higher values than the wild-type TIR+80.

Two gain-of-function mutant forms of TIR+80, W84A, and D136E137NQ were cloned into the pTA7002 vector and tested in $N$. benthamiana plants to see whether the cell death they induced was still dependent on EDS1, SGT1, and HSP90. Indeed, cell death elicited by expression of these mutant forms was dependent on all of these genetic components (Fig. 3A).

\section{DISCUSSION}

Previous data have suggested that the TIR domain of TIRNB-LRR resistance proteins is involved in initiating a signal transduction pathway leading to apoptosis (Frost et al. 2004; Weaver et al.2006; Zhang et al. 2004). Here, we show that transient expression RPS4 derivatives without NB-ARC domain $(\mathrm{TIR}+45 / \mathrm{TIR}+80)$ in either tobacco leaves or Arabidopsis stable transgenic lines causes cell death. Expression of "NBARC-less" constructs of RPP1A or At $4 \mathrm{~g} 19530$ proteins also led to autonecrosis in tobacco leaves. Not all of the TIR+45 homologous constructs are capable of cell death induction. In some instances, this might be due to a low amount of protein accumulation, exemplified by At4g19520 and $N$. However, the $R P P 2 B$ (At4g19510) TIR+45 construct did not induce cell death despite a relatively high level of protein accumulation. Arabidopsis Col-0 RPP2B requires RPP2A (At4g19500) in order to confer resistance against Hyaloperonospora parasitica race cala2 (Sinapidou et al. 2004). The lack of cell death
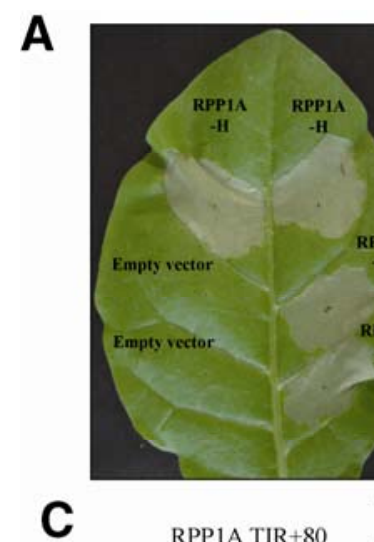

C

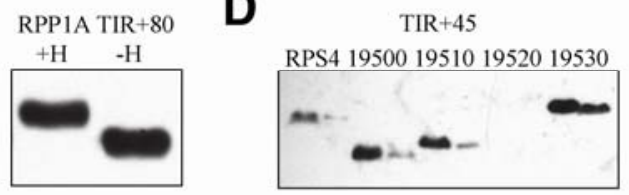

B
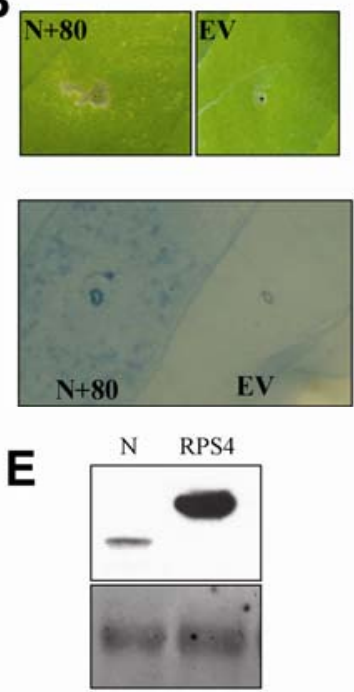

Fig. 4. Expression of TIR+80/TIR+45 constructs from various resistance proteins induces cell death. A, Tobacco leaves were infiltrated with Agrobacterium spp. carrying marked TIR+80 (RPP1A; left picture) or TIR+45 constructs (right picture). RPP1A and At4g19530 derivatives induced cell death. Pictures were taken 3 to 4 days postinfiltration. B, Expression of the N TIR+80 fragment in tobacco leaves induces weak cell death. Lower picture shows the trypanblue-stained tobacco leaf to visualize dead cells. EV = empty vector control. C, Expression of RPP1A TIR+80:HA with (+H) and without (-H) hydrophobic $\mathrm{N}$-terminal fragment. Protein blot was probed with anti-hemagglutinin (HA)-HRP conjugated antibody. D, Western blot showing the lack of expression of the At4g19520 TIR+45 fragment. All other proteins were detected with an anti-HA-HRP conjugated antibody. Two sets of samples, collected $24 \mathrm{~h}$ (weaker signal) and $48 \mathrm{~h}$ (stronger signal) after Agrobacterium infiltration, loaded on the gel in D. E, N TIR+80 accumulates to a substantially lower amount then RPS4 TIR+80. Lower panel shows Ponceau S staining of membrane as an equal protein-loading control. Visualized bands represent the large subunit of rubisco. Expressed proteins were detected as in C and D. 
induced by RPP2B might be due to a unique mode of cooperation between these proteins to provide immunity. Our data are consistent with the idea that the function of the NB-ARC domain in $\mathrm{R}$ proteins might be to provide an energy-dependent conformational change in order to expose the N-terminus for recruitment of components required for cell death signaling. Ploop-dependent conformational change of the $\mathrm{CC}$ containing $\mathrm{R}$ protein Rx has previously been inferred (Moffett et al. 2002). Mutations in the ATP-binding motif of many resistance proteins abolish their function and lead to plant susceptibility to corresponding pathogens (Dinesh-Kumar et al. 2000; Tao et al. 2000; Tornero et al. 2002a). An energy-driven conformational change of APAF1, stimulated by binding cytochrome $\mathrm{C}$ to WD40 repeats, allows N-terminal CARD domains to selfinteract, a key step in apoptosome assembly (Hu et al. 1999). The intact nucleotide binding motif in the tobacco resistance protein $\mathrm{N}$ is necessary for its oligomerization, occurring in the presence of the viral elicitor P50 (Mestre and Baulcombe 2006). How exactly the activities of $R$ proteins are negatively regulated is not clear. Transient expression of RPS2, RPS4, or $R P P 1 A$ in $N$. benthamiana leaves causes cell death, indicating that either at least a portion of the accumulated protein is in an active conformation or additional components are necessary to block activity of these proteins (Day et al. 2005; Weaver et al. 2006; Zhang et al. 2004). Indeed, co-expression of RIN4 with RPS2 in N. benthamiana leaves abolishes RPS2-induced cell death (Day et al. 2005).
Plant signal transduction pathways leading to cell death during resistance responses remain mysterious. Signaling initiated by TIR R proteins is compromised by mutations in the EDS1 gene (Aarts et al. 1998; Falk et al. 1999). EDS1 resembles lipases and interacts with itself and with other lipase-like proteins, PAD4 and SAG101 (Feys et al. 2001, 2005). EDS1 also controls cell death elicited by transient expression of RPS4 and its truncated derivatives (Zhang et al. 2004). Dependence of the TIR+80-mediated cell death on EDS1 indicates that such a cell death mirrors that observed during pathogen ingress rather than being due to nonspecific interference of TIR +80 with cellular metabolism or cell integrity. The TIR+80-induced cell death is also dependent on SGT1 and HSP90. This is consistent with findings which suggest an additional role for SGT1 in plant resistance other than only to cotrol the abundance of $\mathrm{R}$ proteins in the cell or their folding (Holt et al. 2005). Indeed, interactions of SGT1 with components of the putative proteasome lid prompted speculation that SGT1 participates in the removal of negative regulators of apoptosis (Azevedo et al. 2002; Holt et al. 2005). Identification of F-box and U-box proteins as necessary components of signaling pathways in plant disease resistance and cell death promotion is consistent with this view, although the targets degraded by these proteins are unknown (González-Lamothe et al. 2006; Suk Kim and Delaney 2002; Yang et al. 2006).

The crystal structure of the TIR domain from the TLR1, TLR2, and IL-1RAPL shows a similar basic structure, one that

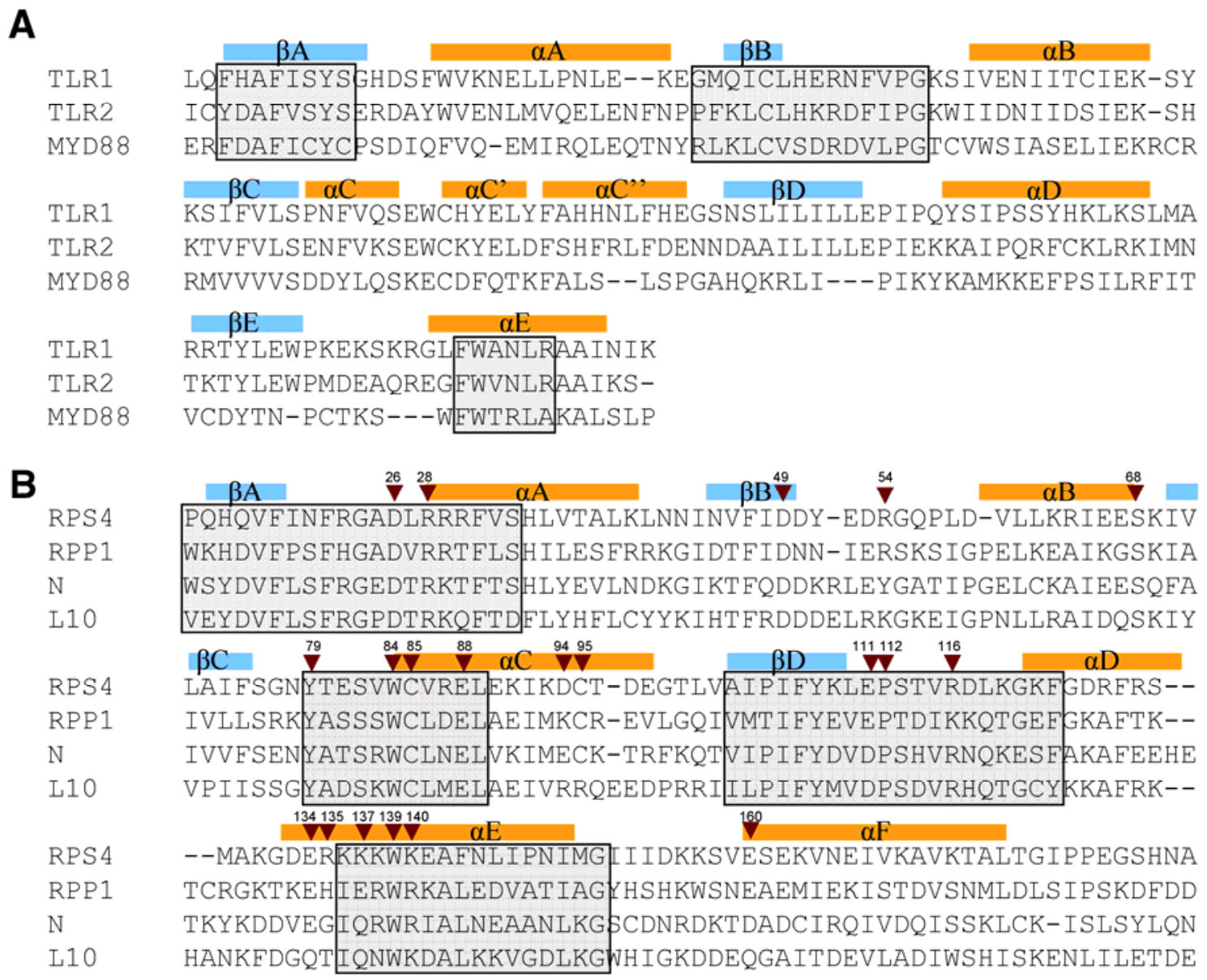

Fig. 5. Amino acid alignment of TIR (toll interleukin 1 receptor) domain sequences. A, Alignment of TIR domains from TLR1, TLR2, and MYD88. Blue and orange bars represent $\beta$-sheets and $\alpha$-helices according to Xu and associates (2000). B, Alignment of plant TIR domains from several resistance proteins. Blue and orange bars represent $\beta$-sheets and $\alpha$-helices according to $\mathrm{Xu}$ and associates (2000). Secondary structures were predicted by numerous programs online. Predicted helix adjacent to the C-terminus of $\alpha \mathrm{E}$ was named $\alpha \mathrm{F}$. Note the lack of $\beta \mathrm{E}$. Arrowheads indicate amino acids that were substituted and numbers above them mark position in the RPS4 TIR domain. Light-gray boxes mark conserved regions in animal TIR domains as defined by Slack and associates (2000) or in plant TIR domain as defined by Meyers and associates (2002). 
is represented by a five-stranded $\beta$-sheet surrounded by $\alpha$-helices (Xu et al. 2000). Different surfaces of the TLR TIR domain have been implicated in dimerization and binding of either Myd88 or Mal adaptors (Dunne et al. 2003; Xu et al. 2000). Many mutations that affect signaling from the interleukin receptor IL1-R or TLR interfere with binding of adaptor proteins (Takeda and Akira 2005). The secondary structure of the plant TIR domain resembles the one in TLR, although it seems that $\beta$-strand $\mathrm{E}$ may be missing. The molecular consequences of such a difference are unknown. At this moment, it is unclear whether the RPS4 TIR domain is sufficient to signal cell death because the construct expressing the TIR domain only does not produce a detectable amount of protein (Zhang et al. 2004). However, we have not observed cell death elicitation by expression of only the TIR domain from either $\mathrm{N}$ or RPP1A (Mestre and Baulcombe 2006; Weaver et al. 2006). This suggests that a part of the sequence between the TIR domain and the NB motif is also important for signaling with the TIR domain itself. The requirement of $\mathrm{C}$-terminal sequence for TIR-domain-mediated cell death signaling is corroborated by the E160A substitution which abolishes cell death even though the protein still accumulates well (Fig. 5). These results suggest that this predicted $\alpha$-helix forms a functional signaling entity with the TIR domain itself.

An amino acid alignment of plant TIR domains has revealed that nearly one-third of residues display a high level

Table 1. List of mutations introduced individually into the TIR domain of RPS4 and their impact on cell death ${ }^{\mathrm{a}}$

\begin{tabular}{|c|c|}
\hline Position of mutation & Strength of cell death \\
\hline TIR80 (control) & ++ \\
\hline D26A & - \\
\hline R28E & - \\
\hline D49A & - \\
\hline D49D50/AA & + \\
\hline D50E52D53/NQN & - \\
\hline $\mathrm{R} 54 \mathrm{~N}$ & - \\
\hline S68A & - \\
\hline Y79A & - \\
\hline Y79F & - \\
\hline W84A & ++++ \\
\hline C85A & - \\
\hline E88A & - \\
\hline D94N & ++ \\
\hline C95A & ++ \\
\hline D97E98/QN & ++++ \\
\hline D97E98/KK & ++ \\
\hline E111K & ++++ \\
\hline P112A & - \\
\hline $\mathrm{P} 112 \mathrm{~K}$ & - \\
\hline S113T114/EE & - \\
\hline R116A & - \\
\hline M129A130 & - \\
\hline E134A & ++++ \\
\hline E134K & ++++ \\
\hline R135E & - \\
\hline D133E134/NQ & ++++ \\
\hline K137E & - \\
\hline K136K137K138 & - \\
\hline W139A & - \\
\hline W139F & - \\
\hline K140N & - \\
\hline E160A & - \\
\hline
\end{tabular}

${ }^{\text {a }}$ Strength of the cell death was visually assessed by comparing the onset of cell death and an extent of collapsed tissue between mutated forms of TIR+80 and the wild type. The following scoring system was used: lack of cell death, -; cell death similar to wild type, ++; cell death weaker then wild type + ; and cell death stronger then wild type, ++++. Mutations showing increased cell death activity are in bold. Double or triple substitutions are separated from the original amino acid sequence by forward slash (/); $\Delta$ indicates deletion of amino acids. of conservation. Three regions (box 1 , box2, and box3) which have been shown to be important for TLR and IL1-R signaling display a high degree of identity in plant TIR domains. More detailed analyses of plant TIR domains have revealed the presence of four rather then three conserved regions, named TIR1-4 (Meyers et al. 2002, 2003). It has been suggested that these boxes play an important role in homotypic interactions between TIR domains (Slack et al. 2000). Interactions between the TIR domain of TLR and Myd88 or MAL are crucial for recruitment of IRAKs and TRAF6 into an activated receptor complex (Wu and Arron 2003). It seems also

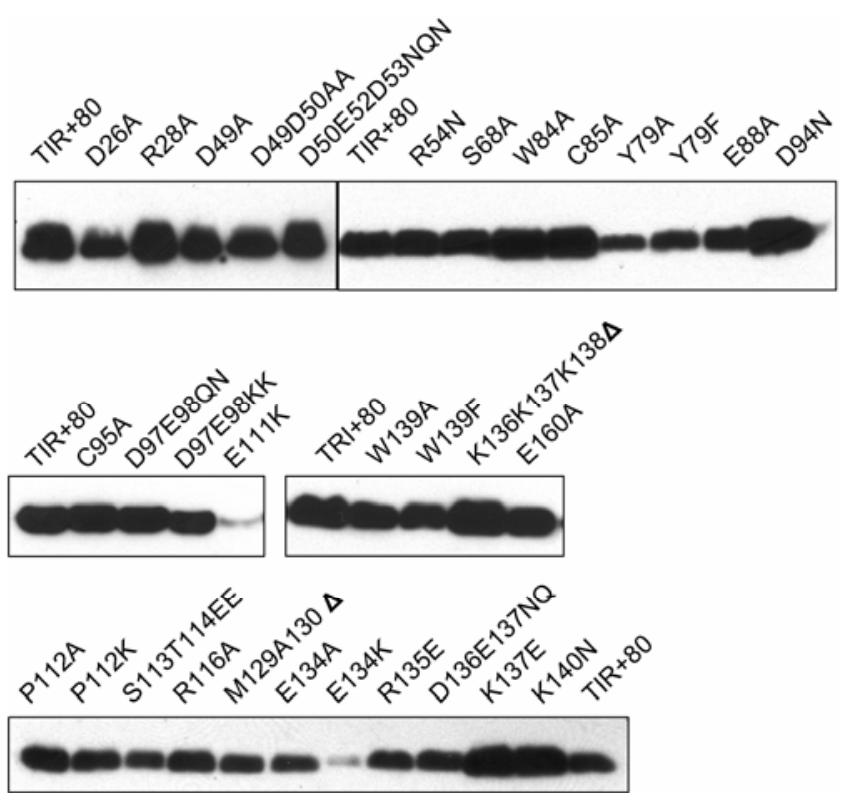

Fig. 6. Expression of mutant forms of the RPS4 TIR+80. Substituted forms of the TIR +80 fragment were delivered into a tobacco leaf by Agrobacterium spp. Leaf samples were taken $40 \mathrm{~h}$ after the Agrobacterium spp. infiltration or prior the uniform tissue collapse (cell death) of the infiltrated area. Expressed proteins were visualized by probing the blot with an anti-hemagglutinin-HRP conjugate. Most of the substitutions were expressed to a level similar to wild-type TIR+80. Low amounts of E111K and $\mathrm{E} 134 \mathrm{~K}$ were reproducibly observed and correlated with strong cell death elicitation by these mutant forms. Equal protein loading was confirmed by staining membranes with the Ponceau S dye (not shown).

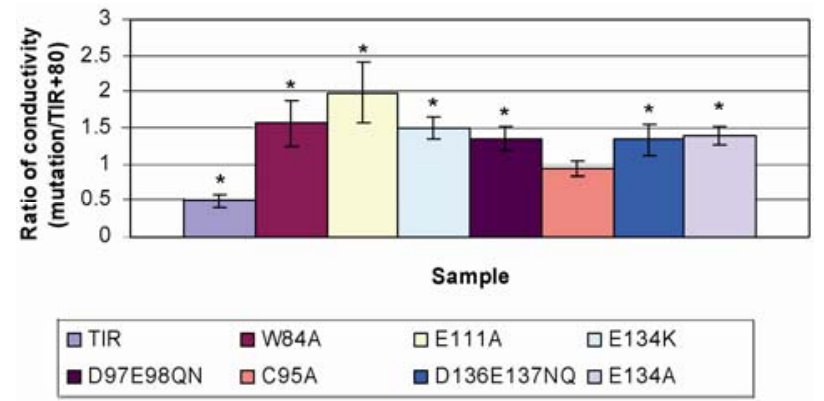

Fig. 7. Several substitutions in the RPS4 TIR (toll interleukin 1 receptor) domain exhibit gain of function phenotype. Agrobacterium spp. harboring corresponding mutated derivatives of TIR +80 were infiltrated in half of a tobacco leaf. Second half of the same leaf was infiltrated with the Agrobacterium strain carrying the wild-type TIR +80 fragment. Sample and control leaf disks were taken from symmetrical position on the same leaf and washed, and conductivity of the water measured $2 \mathrm{~h}$ later. Shown are means of conductivity ratios sample:wild type TIR +80 of at least six independent measurements. Error bars represent the standard deviation. Star above bars indicates statistically significant differences at $P<0.03$. C95A did not change activity (ratio $\approx 1$ ). TIR is an inactive control (ratio $<1)$. 
that interactions between TIR domains are essential for receptor dimerization driven by the recognition of elicitor (Takeda and Akira 2005), a feature also observed for the $\mathrm{N}$ protein (Mestre and Baulcombe 2006). Our attempts to coimmunoprecipitate HA- and Myc-tagged TIR+80s from either RPS4 or RPP1A were unsuccessful (not shown). This might be due to extra $\mathrm{C}$-terminal sequence present in the TIR +80 protein or an instability of such a complex. We cannot rule out the possibility that the RPS4 TIR domain interacts with the TIR domain of other R proteins upon activation.

Site-directed mutagenesis of the RPS4 TIR domain followed by transient cell death assays have revealed that substitution of conserved amino acids in TIR1, TIR2, TIR3, and TIR4 boxes led to loss of function. Substitutions occupying a homologous position in $\mathrm{N}$ and RPS4 produced similar results. (DineshKumar et al. 2000; Mestre and Baulcombe 2006). Such similarities emphasize the universal character of cell death initiation by TIR R proteins.

Interestingly, a few amino acid substitutions potentiated cell death activity of TIR +80 . These gain of function substitutions are located in conserved boxes TIR2 (W84A) and TIR3 $(\mathrm{E} 111 \mathrm{~K})$ and in loosely conserved sequences between boxes TIR2-3 (D97E98/NQ) and TIR3-4 (E134A and E134K). It is not clear how these mutations may elevate the activity of TIR+80. We hypothesize that the conformational change in the TIR domain exerted by such substitutions increases interactions with an unknown factor that is required for initiation of cell death. Indeed, box TIR4 is located in the $\alpha \mathrm{E}$ helix and this helix has been suggested to be engaged in protein-protein interactions (Radons et al. 2003). The sequence of this helix shows a low level of identity between plant TIR domains but its distinct feature is the accumulation of positively and negatively charged residues. Conceivably, the conformation of this region might determine the active or inactive status of the $R$ protein to signal cell death. Such a hypothesis might be addressed in the future by obtaining the crystal structure of the RPS4 TIR domain and corresponding gain-of-function mutant forms and might be helped by the identification of proteins that interact with the TIR domain.

\section{MATERIALS AND METHODS}

\section{Construct preparation.}

Deletion constructs of RPS4 and TIR +80 or TIR +45 homologous sequences from other genes were prepared by amplification of corresponding fragments using polymerase chain reaction (PCR). Primers used for amplification were tailed with appropriate restriction sites. PCR products were then digested with corresponding restriction enzymes and cloned into ClaI/BamHI sites of the pBIN19:HA binary vector (Zhang et al. 2004). For cloning into pER8 or pTA7002 vectors, the TIR+80:HA of RPS4 was amplified with XhoI and NheI sites at the $5^{\prime}$ and $3^{\prime}$ end, respectively, and cloned into XhoI/SpeI sites of vectors. All primer sequences used in the preparation of constructs will be provided upon request.

\section{Agrobacterium spp. transient assay, silencing, and trypan blue staining.}

The transient expression of constructs was done essentially as described in (Zhang et al. 2004) but Agrobacterium spp. were suspended in the ASB buffer $(5 \mathrm{mM} \mathrm{MgCl} 2 ; 5 \mathrm{mM}$ morpholineethanesulfonic acid (MES), pH 5.6; and $100 \mu \mathrm{M}$ acetosyringone). Silencing of $S G T 1, E D S 1$, and $H S P 90$ was performed as reported earlier (Zhang et al. 2004). A maximum of two leaves per silenced plant were tested. Trypan blue staining was done according to Zhang and associates (2004).

\section{Arabidopsis stable transformation.}

Arabidopsis stable transformations were done by following the standard approach (Clough and Bent 1998). Transgenic lines were selected on solid MS medium with hygromycin at a concentration of $40 \mu \mathrm{g} / \mathrm{ml}$.

\section{Ion leakage measurements and Agrobacterium spp. dilution assay.}

To measure the ion leakage caused by the progressing cell death, Agrobacterium strains carrying corresponding constructs were suspended in ASB $\left(2 \mathrm{mM} \mathrm{MgCl}_{2}\right.$ and $2 \mathrm{mM}$ MES, $\mathrm{pH}$ 5.6) and infiltrated into half of a tobacco leaf. The other half was infiltrated with the reference construct $(35 S:: T I R+80: H A)$. At 36 to $48 \mathrm{~h}$ postinfiltration, two leaf disks $\left(1.5 \mathrm{~cm}^{2}\right)$ were taken per leaf sector, briefly washed in $50 \mathrm{ml}$ of water, and put into $2 \mathrm{ml}$ of water with an addition of Silwet-L77 (0.0005\%) and vacuum infiltrated for $2 \mathrm{~min}$. The vacuum was gently released and leaf disks were visually inspected for uniform infiltration. Reference samples were taken always from symmetrical sectors of the same leaf to minimize the variation due to the position on a leaf from which samples were punched. Conductivity of the solution was measured after infiltration (time 0 ) and $2 \mathrm{~h}$ later. Conductivity was recorded with a conductivity meter. For the Agrobacterium dilution assay, strains harboring corresponding constructs were diluted in ASB buffer to optical densities 0.1, 0.025, 0.00625, and 0.00155. Diluted suspensions of Agrobacterium spp. were hand infiltrated into individual panels of a tobacco leaf. The other half of the same leaf was infiltrated with an Agrobacterium strain carrying wildtype $\mathrm{TIR}+80$ diluted to the same density.

\section{Protein analysis.}

Leaf tissue samples were collected $48 \mathrm{~h}$ after the Agrobacterium sp. infiltration or just prior to cell death occurring. Leaf disks $(0.7 \mathrm{~cm}$ in diameter, two disks per sample) were ground in liquid nitrogen in an Eppendorf tube and $100 \mu$ of the loading buffer (120 mM Tris-HCl, pH 6.8; 3\% sodium dodecyl sulfate [SDS]; $100 \mathrm{mM}$ dithiothreitol; $12 \%$ glycerol; and Bromophenol blue at $0.04 \mathrm{mg} / \mathrm{ml}$ ) was added. Samples were briefly vortexed and incubated for $5 \mathrm{~min}$ at $95^{\circ} \mathrm{C}$. Samples were centrifuged for $5 \mathrm{~min}$ and 10 to $15 \mu \mathrm{l}$ of supernatant was loaded on $12 \% \mathrm{SDS} /$ polyacrylamide protein gel. After electrophoresis, proteins were blotted onto an ECL nitrocellulose membrane (Amersham-Pharmacia, Piscataway, NJ, U.S.A.). Expressed proteins were visualized by appropriate HRP conjugated antibodies (HA, clone 3F10; Roche) and Supersignal West Pico (or Femto or a mix of both of them) Chemiluminescent Substrates (Pierce, Rockford, IL, U.S.A.). Antibodies were diluted according to the manufacturer's recommendations.

\section{Site-directed mutagenesis.}

The cDNA fragment of RPS4 TIR+80 was prepared by PCR using the RPS4 full-length cDNA as a template (Zhang et al. 2004). Primers used in the PCR contained ClaI and BamHI restriction sites, respectively. The amplified fragment was cloned into pGEMT-easy (Promega Corp., Madison, WI, U.S.A.) and served as a template for an in vitro mutagenesis. Mutations in the RPS4 TIR+80 were introduced following instructions to QuickChange XL Site-Directed Mutagenesis Kit (Stratagene, La Jolla, CA, U.S.A.). Substituted forms of TIR+80 were recloned into pBin19:HA. The sequences of all primers used will be available upon request.

\section{Dexamethasone and estradiol treatment.}

Estradiol and dexamethasone were dissolved in ethanol and spread on the surface of solid MS medium to a final concentration of the inducer in total volume of medium $(25 \mathrm{ml})$ of 40 
$\mu \mathrm{M}$. Plates were left open until the ethanol evaporated and kept at room temperature at least $24 \mathrm{~h}$ to facilitate diffusion of chemicals throughout the medium. Two-week-old seedlings grown on the solid MS medium supplemented with hygromycin were transplanted onto a fresh plate with inducers and grown further until visual symptoms appeared (2 to 5 days). To analyze protein expression, seedlings were collected 24,48 , and $72 \mathrm{~h}$ after transplantation on the expression induction medium. For infiltrations, dexamethasone was diluted in water to the final concentration of $40 \mu \mathrm{M}$.

\section{Sequence alignment.}

Alignment of amino acid sequences of TIR domains was done using ClustalX (Thomson et al. 1997). Alignment was manually corrected and displayed in GeneDoc (Nicholas et al. 1997). Secondary structure predictions were conducted by various programs accessible online.

\section{ACKNOWLEDGMENTS}

We thank P. Mestre for providing cDNA of the $N$ gene, M. Smoker for reading and correcting the manuscript, and $\mathrm{K}$. Sohn for discussion and advice on ion leakage assay. This work was supported by the Biotechnology and Biological Science Research Council grant P15093. The work in the Sainsbury Laboratory is supported by the Gatsby Charitable Foundation.

\section{LITERATURE CITED}

Aarts, N., Metz, M., Holub, E., Staskawicz, B. J., Daniels, M. J., and Parker, J. E. 1998. Different requirements for EDS1 and NDR1 by disease resistance genes define at least two $\mathrm{R}$ gene-mediated signaling pathways in Arabidopsis. Proc. Natl. Acad. Sci. U.S.A. 95:1030610311.

Aoyama, T., and Chua, N. H. 1997. A glucocorticoid-mediated transcriptional induction system in transgenic plants. Plant J. 11:605-612

Axtell, M. J., and Staskawicz, B. 2003. Initiation of RPS2-specified disease resistance in Arabidopsis is coupled to the AvrRpt2-directed elimination of RIN4. Cell 112:369-377.

Azevedo, C., Sadanandom, A., Kitagawa, K., Freialdenhoven, A., Shirasu, K., and Schulze-Lefert, P. 2002. The RAR1 interactor SGT1, an essential component of $R$ gene-triggered disease resistance. Science 295:2073-2076.

Bieri, S., Mauch, S., Shen, Q. H., Peart, J., Devoto, A., Casais, C., Ceron, F., Schulze, S., Steinbiss, H. H., Shirasu, K., and Schulze-Lefert, P. 2004. RAR 1 positively controls steady state levels of barley MLA resistance proteins and enables sufficient MLA6 accumulation for effective resistance. Plant Cell 16:3480-3495.

Botella, M. A., Parker, J. E., Frost, L. N., BittnerEddy, P. D., Beynon, J. L., Daniels, M. J., Holub, E. B., and Jones, J. D. G. 1998. Three genes of the Arabidopsis RPP1 complex resistance locus recognize distinct Peronospora parasitica avirulence determinants. Plant Cell 10:1847-1860.

Clough, S. J., and Bent, A. F. 1998. Floral dip: A simplified method for Agrobacterium-mediated transformation of Arabidopsis thaliana. Plant J. 16:735-743

Dangl, J. L., and Jones, J. D. G. 2001. Plant pathogens and integrated defence responses to infection. Nature 411:826-833.

Day, B., Dahlbeck, D., Huang, J., Chisholm, S. T., Li, D., and Staskawicz B. J. 2005. Molecular basis for the RIN4 negative regulation of RPS2 disease resistance. Plant Cell 17:1292-1305.

Dinesh-Kumar, S. P., Tham, W. H., and Baker, B. J. 2000. Structure-function analysis of the tobacco mosaic virus resistance gene N. Proc. Natl. Acad. Sci. U.S.A. 97:14789-14794.

Dodds, P. N., Lawrence, G. J., Catanzariti, A., Teh T, Wang C.-I. A., Ayliffe M. A., Kobe, B., and Ellis, J. G. 2006. Direct protein interaction underlies gene-for-gene specificity and co-evolution of the flax L5/L6/L7 resistance genes and flax rust AvrL567 avirulence genes. Proc. Natl. Acad. Sci. U.S.A. 103:8888-8893.

Dunne, A., Ejdebäck, M., Ludidi, P. L., Luke A. J. O'Neill, L. A. J., and Gay, N. J. 2003. Structural complementarity of Toll/interleukin-1 receptor domains in toll-like receptors and the adaptors Mal and MyD88. J. Biol. Chem. 278:41443-41451.

Falk, A., Feys, B. J., Frost, L. N., Jones, J. D. G., Daniels, M. J., and Parker, J. E. 1999. EDS1, an essential component of R gene-mediated disease resistance in Arabidopsis has homology to eukaryotic lipases. Proc. Natl. Acad. Sci. U.S.A. 96:3292-3297.
Feys, B. J., Moisan, L. J., Newman, M. A., and Parker, J. E. 2001. Direct interaction between the Arabidopsis disease resistance signaling proteins, EDS1 and PAD4. EMBO (Eur. Mol. Biol. Organ.) J. 20:54005411.

Feys, B. J., Wiermer, M., Bhat, R. A., Moisan, L. J., Medina-Escobar, N., Neu, C., Cabral, A., and Parker, J. E. 2005. Arabidopsis SenescenceAssociated Gene 101 stabilizes and signals within an EDS1 complex in plant innate immunity. Plant Cell 17:2601-2613.

Frost, D., Way, H., Howles, P., Luck, J. E., Manners, J., Hardham, A., Finnegan, J., and Ellis, J. 2004. Tobacco transgenic for the flax rust resistance gene $L$ expresses allele-specific activation of defense responses. Mol. Plant-Microbe Interact. 17:224-232.

González-Lamothe, R., Tsitsigiannis, D. I., Ludwig, A., Panicot, M., Shirasu, K., and Jones J. D. G. 2006. The U-box protein CMPG1 is required for efficient activation of defense mechanisms triggered by multiple resistance genes in tobacco and tomato. Plant Cell 18:1084-1098.

Hammond-Kosack, K. E., and Jones, J. D. G. 1997. Plant disease resistance genes. Annu. Rev. Plant Physiol. Plant Mol. Biol. 48:575-607.

Holt, B. F., III, Belkhadir, Y., and Dangl, J. L. 2005. Antagonistic control of disease resistance protein stability in the plant immune system. Science 309:929-933.

Hu, Y., Benedict, M., Ding, L., and Nunez, G. 1999. Role of cytochrome $c$ and dATP/ATP hydrolysis in Apaf-1-mediated caspase-9 activation and apoptosis. EMBO (Eur. Mol. Biol. Organ.) J. 18:3586-3595.

Hubert, D. A., Tornero, P., Belkhadir, Y., Krishna, P., Takahashi, A., Shirasu, K., and Dangl, J. L. 2003. Cytosolic HSP90 associates with and modulates the Arabidopsis RPM1 disease resistance protein. EMBO (Eur. Mol. Biol. Organ.) J. 22:5679-5689.

Jones, D. A., and Takemoto, D. 2004. Plant innate immunity—direct and indirect recognition of general and specific pathogen-associated molecules. Curr. Opin. Immunol. 16:48-62.

Leister, T. R., and Katagiri, F. 2000. A resistance gene product of the nucleotide binding site-leucine rich repeats class can form a complex with bacterial avirulence proteins in vivo. Plant J. 22:345-354.

Leister, T. R., Dahlbeck, D., Day, B., Li, Y., Chesnokova, O., and Staskawicz, B. J. 2005. Molecular genetic evidence for the role of SGT1 in the intramolecular complementation of Bs2 protein activity in Nicotiana benthamiana. Plant Cell 17:1268-1278.

Mackey, D., Holt, B. F., Wiig, A., and Dangl, J. L. 2003. RIN4 interacts with Pseudomonas syringae type III effector molecules and is required for RPM1-mediated resistance in Arabidopsis. Cell 108:743-754.

Mestre, P., and Baulcombe, D. C. 2006. Elicitor-mediated oligomerization of the tobacco N disease resistance protein. Plant Cell 18:491-501.

Meyers, B. C., Morgante, M., and Michelmore, R. W. 2002. TIR-X and TIR-NBS proteins: Two new families related to disease resistance TIRNBS-LRR proteins encoded in Arabidopsis and other plant genomes. Plant J. 32:77-92.

Meyers, B. C., Kozik, A., Griego, A., Kuang, H., and Michelmore, R. W. 2003. Genome-wide analysis of NBS-LRR-encoding genes in Arabidopsis. Plant Cell 15:809-834.

Moffett, P., Farnham, G., Peart, J. R., and Baulcombe, D. C. 2002. Interaction between domains of a plant NBS-LRR protein in disease resistance-related cell death. EMBO (Eur. Mol. Biol. Organ.) J. 21:45114519.

Nicholas, K. B., Nicholas, H. B., Jr., and Deerfield, D. W., II. 1997. GeneDoc: Analysis and visualization of genetic variation. Embnet News 4:1-4.

Oldroyd, G. E., and Staskawicz, B. 1998. Genetically engineered broadspectrum disease resistance in tomato. Proc. Natl. Acad. Sci. U.S.A 95:10300-10305

Radons, J., Dove, S., Neumann, D., Altmann, R., Botzki, A., Martin, M. U., and Falk, W. 2003. The interleukin 1 IL-1 receptor accessory protein Toll/IL-1 receptor domain. J. Biol. Chem. 278:49145-49153.

Rairdan, G. J., and Moffett, P. 2006. Distinct domains in the ARC region of the Rx NB-LRR protein mediate LRR binding and inhibition of activation. Plant Cell 18:2082-2093.

Riedl, S. J., Li, W., Chao, Y., Schwarzenbacher, R., and Shi, Y. 2005 Structure of the apoptotic protease-activating factor 1 bound to ADP. Nature 434:926-933.

Shao, F., Golstein, C., Ade, J., Stoutemyer, M., Dixon, J. E., and Innes, R. W. 2003. Cleavage of Arabidopsis PBS1 by a bacterial type III effector. Science 301:1230-1233.

Sinapidou, E., Williams, K., Nott, L., Bahkt, S., Tör, M., Crute, I., BittnerEddy, P., and Beynon, J., 2004. Two TIR:NB:LRR genes are required to specify resistance to Peronospora parasitica isolate Cala2 in Arabidopsis. Plant J. 38:898-909.

Slack, J. L., Schooley, K., Bonnert, T. P., Mitcham, J. L., Qwarnstrom, E. E., Sims, J. E., and Dower, S. K. 2000. Identification of two major sites in the type I interleukin-1 receptor cytoplasmic region responsible for coupling to pro-inflammatory signaling pathways. J. Biol. Chem. 275:4670-4678. 
Suk Kim, H., and Delaney, T. P. 2002. Arabidopsis SON1 is an F-box protein that regulates a novel induced defense response independent of both salicylic acid and systemic acquired resistance. Plant Cell 14:14691482.

Takahashi, A., Casais, C., Ichimura, K., and Shirasu, K. 2003. HSP90 interacts with RAR1 and SGT1, and is essential for RPS2-mediated disease resistance in Arabidopsis. Proc. Natl. Acad. Sci. U.S.A. 100:1177711782.

Takeda, K., and Akira, S. 2005. Toll-like receptors in innate immunity. Int. Immunol. 17:1-14.

Tameling, W. I. L., Elzinga, S. D. J., Darmin, P. S., Vossen, J. H., Takken, F. L. W., Harling, M. A., and Cornelissen, B. 2002. The tomato $R$ gene products 1-2 and Mi-1 are functional ATP binding proteins with ATPase activity. Plant Cell 14:2929-2939.

Tameling, W. I., Vossen, J. H., Albrecht, M., Lengauer, T., Berden, J. A., Haring, M. A., Cornelissen, B. J., and Takken, F. L. 2006. Mutations in the NB-ARC domain of I-2 that impair ATP hydrolysis cause autoactivation. Plant Physiol. 140:1233-1245.

Tao, Y., Yuan, F. H., Leister, R. T., Ausubel, F. M., and Katagiri, F. 2000. Mutational analysis of the Arabidopsis nucleotide binding site-leucinerich repeat resistance gene RPS2. Plant Cell 12:2541-2554.

Thompson, J. D., Gibson, T. J., Plewniak, F., Jeanmougin, F., and Higgins, D. G. 1997. The ClustalX windows interface: Flexible strategies for multiple sequence alignment aided by quality analysis tools. Nucleic Acids Res. 25:4876-4882.

Tornero, P., Chao, R. A., Luthin, W. N., Goff, S. A., and Dangl, J. 2002a. Large-scale structure-function analysis of the Arabidopsis RPM1 disease resistance protein. Plant Cell 14:435-450.

Tornero, P., Merritt, P., Sadanandom, A., Shirasu, K., Innes, R. W., and Dangl, J. L. 2002b. RAR1 and NDR1 contribute quantitatively to disease resistance in Arabidopsis, and their relative contributions are dependent on the R gene assayed. Plant Cell 14:1005-1015.

Van Benntem, S de la F., Vossen, J. H., de Vries, K. J., van Wees, S., Tameling, W. I. L., Dekker, H. L, de Koster, C. G., Haring, M. A., Takken, F. L. W., and Cornelissen, B. J. C. 2005. Heat shock protein 90 and its co-chaperone protein phostphatase 5 interact with distinct regions of the tomato I-2 disease resistance protein. Plant J. 43:284298

Van der Biezen, E. A., and Jones, J. D. G. 1998a. The NB-ARC domain: A novel signalling motif shared by plant resistance gene products and regulators of cell death in animals. Curr. Biol. 8:226-227.

Van der Biezen, E. A., and Jones, J. D. G. 1998b. Plant disease-resistance proteins and the gene-for-gene concept. Trends Biochem. Sci. 23:454456.

Weaver, L. M., Swiderski M. R., Li, Y., and Jones, J. D. G. 2006. The Arabidopsis thaliana TIR-NB-LRR R protein, RPP1A; protein localization and constitutive activation of defence by truncated alleles in tobacco and Arabidopsis. Plant J. 47:829-840.

Wu, H., and Arron J. R. 2003. TRAF6, a molecule bridge spanning adaptive immunity, innate immunity and osteoimmunology. BioEssays 25:1096-1105.

Xu, Y., Tao, X., Shen, B., Horng, T., Medzhitov, R., Manley, J. L., and Tong, L. 2000. Structural basis for signal transduction by the Toll/interleukin-1 receptor domains. Nature 408:111-115.

Yang, C., González-Lamothe, R., Ewan, R. A., Rowland, O., Yoshioka, H., Shenton, M., Ye, H., O’Donnell, E., Jones, J. D. G., and Sadanandom, A. 2006. The E3 ubiquitin ligase activity of Arabidopsis plant UBOX17 and its functional tobacco homolog ACRE276 are required for cell death and defense. Plant Cell 18:1084-1098.

Yang, S., and Hua, J. 2004. A haplotype-specific resistance gene regulated by BONZAI1 mediates temperature-dependent growth control in Arabidopsis. Plant Cell 16:1060-1071.

Zhang, Y., Dorey, S., Swiderski, M., and Jones, J. D. G. 2004. Expression of RPS4 in tobacco induces an AvrRps4-independent HR that requires EDS1, SGT1 and HSP90. Plant J. 40:213-224.

Zuo, J., Niu, Q. W., and Chua, N. H. 2000. An estrogen receptor-based transactivator XVE mediates highly inducible gene expression in transgenic plants. Plant J. 24:265-273

\section{AUTHOR-RECOMMENDED INTERNET RESOURCES}

Advanced Protein Secondary Structure Prediction server: www.imtech.res.in/raghava/apssp2

Jpred 3 server: www.compbio.dundee.ac.uk/ www-jpred

National Resource for Biomedical Supercomputing (NRBSC) website: www.psc.edu/biomed/genedoc

PredictProtein server: www.predictprotein.org 